Draft Version OCtOBER 29, 2018

Preprint typeset using LATEX style emulateapj v. 4/12/04

\title{
ANGULAR MOMENTUM TRANSPORT IN ACCRETION DISKS AND ITS IMPLICATIONS FOR SPIN ESTIMATES IN BLACK HOLE BINARIES
}

\author{
Chris Done ${ }^{1}$ and Shane W. Davis ${ }^{2,3}$ \\ Draft version October 29, 2018
}

\begin{abstract}
The accretion flow in the disk dominated state of black hole binaries has peak temperature and luminosity which vary together in such a way as to indicate an approximately constant emitting area. The association of this with the last stable orbit gives one of the few ways to estimate spin when the mass of the black hole is known. However, deriving this radius requires knowledge of how the disk spectrum is modified by radiative transfer through the vertical structure of the disk, as well as special and general relativistic effects on the propagation of this radiation. Here we investigate the extent to which differences in vertical structure change the derived disk spectra by calculating these for a range of different stress prescriptions. We find that at a given mass accretion rate the spectra are almost identical for accretion rates of $L / L_{E d d} \lesssim 0.1$. The spectra are remarkably similar even up to the highest luminosities considered $\left(L / L_{E d d} \sim 0.6\right)$ as long as the stresses do not dissipate more than about 10 per cent of the gravitational energy above the effective photosphere. This is exceeded only by classic alpha disks with $\alpha \gtrsim 0.1$, but these models give spectral variation which is incompatible with existing data. Therefore, we conclude that disk spectral modelling can place interesting constraints on angular momentum transport, but still provide a robust estimate of the spin of the black hole.
\end{abstract}

Subject headings: accretion, accretion disks — black hole physics — X-rays:binaries

\section{INTRODUCTION}

Black hole spin is difficult to measure as it gives a strong signature only very close to the event horizon. Currently the only way to probe this is via luminous accretion flows, which light up the regions of dramatically curved spacetime close to the event horizon. However, to use these as a diagnostic of spin and/or to test of Einsteins gravity in the strong-field limit requires knowledge of the velocity and emission structure of this material. Currently, this is best understood for an geometrically thin, optically thick accretion disk, where the material rotates in approximately Keplerian orbits (except at the inner boundary) and the gravitational energy thermalizes to produce a quasi-blackbody spectrum at each radius in the disk (Shakura \& Sunvaev 1973).

Black hole binaries (BHB) can indeed show spectra which look like this simple 'sum of blackbodies', though these are generally accompanied by a nonthermal tail to higher energies whose origin is not well understood. Nonetheless, where this tail carries only a minor fraction of the bolometric power, then the disk model gives a fairly good description of the spectral shape. More compellingly, a sequence of such disk dominated spectra from the same object at different mass accretion rates shows that the bolometric disk luminosity (spanning factors of 10-50) relates to the maximum temperature as $L_{\text {bol }} \propto T_{\max }^{4}$ (Kubota et al.2001) , or equivalently, that the disk has an approximately constant inner radius (Ebisawa et al. 1991, 1993). This observation is exactly the behavior predicted by General Relativity, as the last stable orbit forms a fixed size scale for a geometrically

\footnotetext{
1 Department of Physics, University of Durham, South Road, Durham, DH1 3LE, UK

${ }^{2}$ School of Natural Sciences, Institute for Advanced Study, Einstein Drive, Princeton, NJ 08540

3 Chandra Fellow
}

thin disk (see e.g. the review by Done et al. 2007, hereafter DGK07).

However, these disk dominated spectra are surprising in the context of the Shakura-Sunyaev disk models as they are seen at high luminosities, typically $0.1-0.5$ $L_{\text {Edd }}$ (e.g. DGK07). The classic prescription in which the viscous stress is directly proportional to total (gas plus radiation, $P_{\text {tot }}=P_{\text {gas }}+P_{\text {rad }}$ ) pressure, is thermally and viscously unstable where radiation pressure dominates, i.e. for luminosities above $\sim 0.05 L_{\mathrm{Edd}}$ for $\mathrm{BHB}$ (e.g. Lightman 1974; Shakura \& Sunvaev 1976). Above this luminosity, the inner regions of alpha disks exhibit limit cycles, in direct conflict with the observed stability of the disk spectra (e.g. Nowak 1995). Yet the alpha prescription does seem to be able to reproduce the long timescale outburst/quiescence behavior of the outer, gas pressure dominated region in the disk instability model (see e.g. the review by Lasota 2001).

Thus it seems likely that the stress is approximately proportional to gas pressure where gas pressure dominates, but that it scales less sensitively with temperature than predicted by radiation pressure when radiation pressure dominates. Ultimately, the question of the stress scaling will be answered by numerical simulations of the Magneto-Rotational Instability (MRI, Balbus \& Hawlev 1991) which forms the physical basis for "effective viscosity". However, this is beyond the scope of current codes as it requires coupled radiativemagneto-hydrodynamic simulations in full general relativity, covering a large radial extent but simultaneously resolving the small scale height of the thin disk!. Until these become available, ad hoc stress scalings are the only way to model the structure of a radiative accretion disk.

The choice of stress prescription can affect the spectrum as well as the stability of the disk as it determines the surface density, and therefore, the overall optical 
depth of the disk. This emission thermalizes to a true blackbody only if the disk is effectively optically thick to absorption at all frequencies. Free-free (continuum) and bound-free (photo-electric edge) absorption opacity both drop as a function of frequency while electron scattering is constant, so the highest energy photons from each radii are less likely to thermalize. The spectrum then becomes a modified blackbody whose frequency dependence can, in general, differ significantly from Planckian. However, the effects of Compton scattering tend to yield a Wien-like tail and the hottest parts of the disk are generally well modelled by a color-corrected (or diluted) blackbody, with effective temperature which is a factor $f_{\text {col }}$ (termed a color-temperature correction) higher than for complete thermalization. The full-disk spectrum is then a sum of modified black bodies, but this can likewise be approximately described by a color-temperature corrected blackbody disk spectrum (Shimura \& Takahara 1995). This color-temperature correction depends on the vertical temperature and density structure of the disk, both of which can only be fully determined once the stress is specified.

Since the observations show to zeroth order that $L \propto$ $T^{4}$, the color-temperature correction must stay approximately constant (the alternative, that it changes in just such a way as to mask any change in innermost extent of the disk, violates Occam's razor). This used to be controversial, with different models of alpha disks (with only $\mathrm{H}$ and $\mathrm{He}$ ) giving different results. Shimura \& Takahara (1995) found that $f_{\text {col }}$ remains relatively constant over a wide range in luminosity, while Merloni et al. (2000) had constant $f_{\text {col only }}$ at $>0.1 L_{\mathrm{Edd}}$, increasing below (Gierliński \& Done 2004, hereafter GD04). Since it is physically rather unlikely that absorption becomes less effective at lower temperatures, the Merloni et al. (2000) result seems rather to be an artifact of the assumed constant vertical density structure. However, both these calculations are now superseded by the models of Davis et al. (2005), which includes fully non-LTE calculations for all abundant elements together with the self-consistent radiative transfer and disk structure. These indeed show that $f_{\text {col }}$ is approximately constant for an alpha disk, but only below temperatures of $\sim 1 \mathrm{keV}$. Above this, the disk becomes so ionized that even photoelectric absorption opacity becomes negligible, so $f_{\text {col }}$ increases markedly (Davis et al. 2005, 2006). A constant $f_{\text {col }}$ can be recovered even at these high temperatures by simply decreasing $\alpha$ to $\sim 0.01$, but this is inconsistent with the $\alpha \sim 0.1$ required in order to match the rapid rise to outburst seen in transient systems (e.g. King et al. 2007), and still gives a disk which should undergo limit cycle oscillations when radiation pressure becomes dominant.

Thus it seems that both spectra and stability of the observed disk spectra in BHBs are inconsistent with an alpha stress prescription. Here we use the methods of Davis et al. (2005) and Davis \& Hubenv (2006) to examine two alternative models, where the stress is directly proportional to gas pressure alone, hereafter called the beta disk, or to the geometric mean of the gas and total pressure, hereafter called the mean disk, (Sakimoto \& Coroniti 1981; Stella \& Rosner 1984; Taam \& Lin 1984; Laor \& Netzer 1989; Merloni 2003). When we fix $\alpha=0.1$ to agree with requirements of the disk instability model, we find that either of these give a better match to the spectral constraints than an alpha prescription as the resulting disk is denser and more optically thick. Thus these alternative stress scalings give disks which are able to maintain a relatively constant $f_{\text {col }}$ even at high temperatures, providing a better match to the observed spectra as well as the stability properties.

While this gives important insight into the approximate form of the MRI stresses, we show that all stress prescriptions considered here (alpha, beta, and mean) give the same $f_{\text {col }}$ below $\sim 1 \mathrm{keV}$, suggesting that disk spectra can be used to give a robust estimate of the inner radius of the disk, hence observationally constraining the black hole spin.

\section{ALTERNATIVE STRESS PRESCRIPTIONS}

The magnetic nature of the viscosity has long been recognized (Shakura \& Sunvaev 1973; Eardley \& Lightman 1975; Ichimaru 1977; Galeev et al. 1979), motivating alternative stress prescriptions (e.g. Sakimoto \& Coroniti 1981). The two most popular are where stress is directly proportional to gas pressure alone (beta disks, Stella \& Rosner 1984) or to the geometric mean of the gas and total pressure, $\sqrt{P_{\text {gas }} P_{\text {tot }}}$ (mean disks, Taam \& Lin 1984; Merloni 2003). Beta disks are stable at all luminosities below the Eddington limit (above which the equations break down as winds and/or advection become important), while the mean disks show limit cycles above $\sim 0.3 L_{\mathrm{Edd}}$ for stellar remnant black holes (e.g. Honma et al. 1991; Merloni \& Navakshin 2006). The mean disk is thus inconsistent with the observation that stable disk spectra exist up to at least $\sim 0.7 L_{\mathrm{Edd}}$ (DKG07), but the onset of the instability is very sensitive to the exact scaling of the stress. For the more general prescription which is proportional to $P_{\text {gas }}^{\mu} P_{\text {tot }}^{1-\mu}$ then the observed stability limit requires $\mu \sim 0.56$ (Kato et al. 1998), which is negligibly different to the mean disk $(\mu=0.5)$ in terms of steady state disk structure.

We use the bhspec code of Davis \& Hubenv (2006) to calculate the self-consistent vertical structure and resulting spectra for three stress prescriptions: alpha, beta, and mean disks. We consider a geometrically thin disk and break it up into a series of logarithmically spaced annuli. We then calculate the one-dimensional vertical structure and radiative transfer in each annulus using the TLUSTY stellar atmosphere code (Hubeny \& Lanz 1995). The total spectrum is then integrated over the disk surface, accounting for the effects of space-time curvature on the photon geodesics. Three parameters determine the structure of each individual annulus: the radiative flux at the disk surface; the surface density, $\Sigma$; and a parameter which determines the strength of the tidal gravity.

Since mechanical energy dissipates directly within the disk "atmosphere", the radiative flux $F$ varies with height $z$ in the disk, requiring an additional assumption to uniquely determine the structure. Following Shakura \& Sunyaev (1973), we assume that the dissipation $d F / d z$ is locally proportional to the density $\rho$. Using the definition of column mass, $d m=-\rho d z$, this is equivalent to

$$
\frac{d F}{d m}=-\frac{2 \sigma T_{\mathrm{eff}}^{4}}{\Sigma}
$$



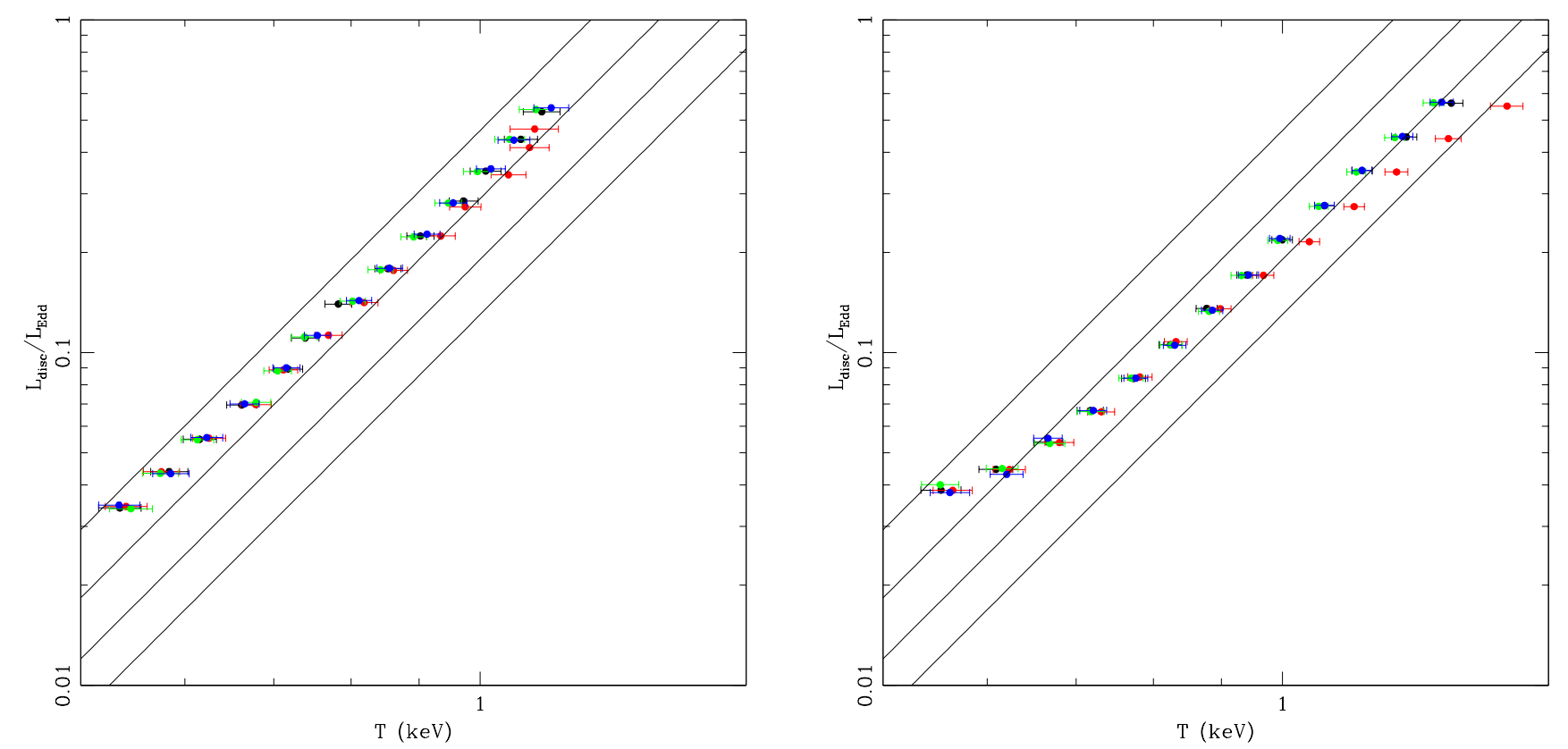

FIG. 1.- Luminosity-Temperature relations derived from fits of bhspec with diskbb as described in 3 The left panel corresponds to fits using a CCD (Swift XRT) response while the right panel utilizes a proportional counter ( $R X T E$ PCA) response. We consider four different stress prescriptions: alpha disks with $\alpha=0.01$ (black) and $\alpha=0.1$ (red), beta disks with $\alpha=0.1$ (blue), and mean disks with $\alpha=0.1$ (green). These all overlap apart from the alpha disks with $\alpha=0.1$ at high luminosity, showing how robust the spectra are to different stress prescriptions as long as the disk remains optically thick. The solid black lines show the $L-T$ relation expected from black hole with $a_{*}=0.5$ for a color-temperature correction of $1.6,1.8,2.0$ and 2.2 from top to bottom, respectively.

where $T_{\text {eff }}$ is the effective temperature of the annulus. Note that the right hand side terms, and therefore $d F / d m$ are independent of height with this assumption. We cannot use the stress prescription to specify a local viscous-like dissipation as this is proportional to some combination of radiation and gas pressure. These pressures are largest at the disk midplane due to hydrostatic equilibrium, so the dissipation is also largest there, and the resulting temperatures are so high as to lead to the disk becoming Rayleigh-Taylor unstable. This would lead to convection, presumably driving the disk structure towards the marginally stable condition where the dissipation is proportional to density. Time and horizontal averages of shearing box simulations (Turner 2004; Hirose et al. 2006) indicate that a somewhat greater fraction of the dissipation occurs at low column near the surface.

We use a vertically integrated disk model to determine the above parameters for each annulus. The tidal gravity is specified by the choice of spacetime and radius, while the radiative flux at the surface is independent of stress prescription for a thin disk (see e.g. Shakura \& Sunvaev 1973). Therefore, we only require a stress prescription to determine the surface density in this model. (Note, however, that the theoretical arguments which motivate the stress prescription may also require modifications to the dissipation profile, a point we will discuss further in \$5.) The surface density may be determined by enforcing angular momentum conservation

$$
\int \tau_{r \phi} d z=\frac{\dot{M} \Omega}{2 \pi} \frac{D(r)}{A(r)},
$$

where $\tau_{r \phi}$ is the accretion stress, $\dot{M}$ is the accretion rate, and $\Omega$ is the Keplerian frequency. The functions $A(r)$ and $D(r)$ (both $\rightarrow 1$ for radii much larger than the last stable orbit) defined in Riffert \& Herold (1995) incorporate the no-torque (stress free) inner boundary condition and parameterize the deviation of general relativistic disk structure from the Newtonian limit.

The integral in eq. (2) presents a computational difficulty since $\tau_{r \phi}$ depends on local variable such as temperature and density for the stress prescriptions considered here. Therefore, the exact computation requires the full vertical structure which, in turn, requires a choice of $\Sigma$. Therefore, an exact solution can only be computed via iteration provided an initial guess for $\Sigma$ (see e.g. Davis et al. 2005). Given the approximations inherent in our stress prescriptions, we instead solve for $\Sigma$ using moderately crude vertical averages to replace $\int \tau_{r \phi} d z$ with $2 H\left\langle\tau_{r \phi}\right\rangle$. Here $H$ is an approximate vertical scale height for the disk determined via the equation of hydrostatic equilibrium

$$
\frac{\partial P_{\text {tot }}}{\partial z}=\rho \Omega^{2} z \frac{C(r)}{B(r)},
$$

where $B(r)$ and $C(r)$ are relativistic factors (again both $\rightarrow 1$ for radii much larger than the last stable orbit) defined in Riffert \& Herold (1995). This is solved by substituting $z \rightarrow H$ and $\partial / \partial z \rightarrow H^{-1}$. We then specify $\left\langle P_{\text {rad }}\right\rangle$ 

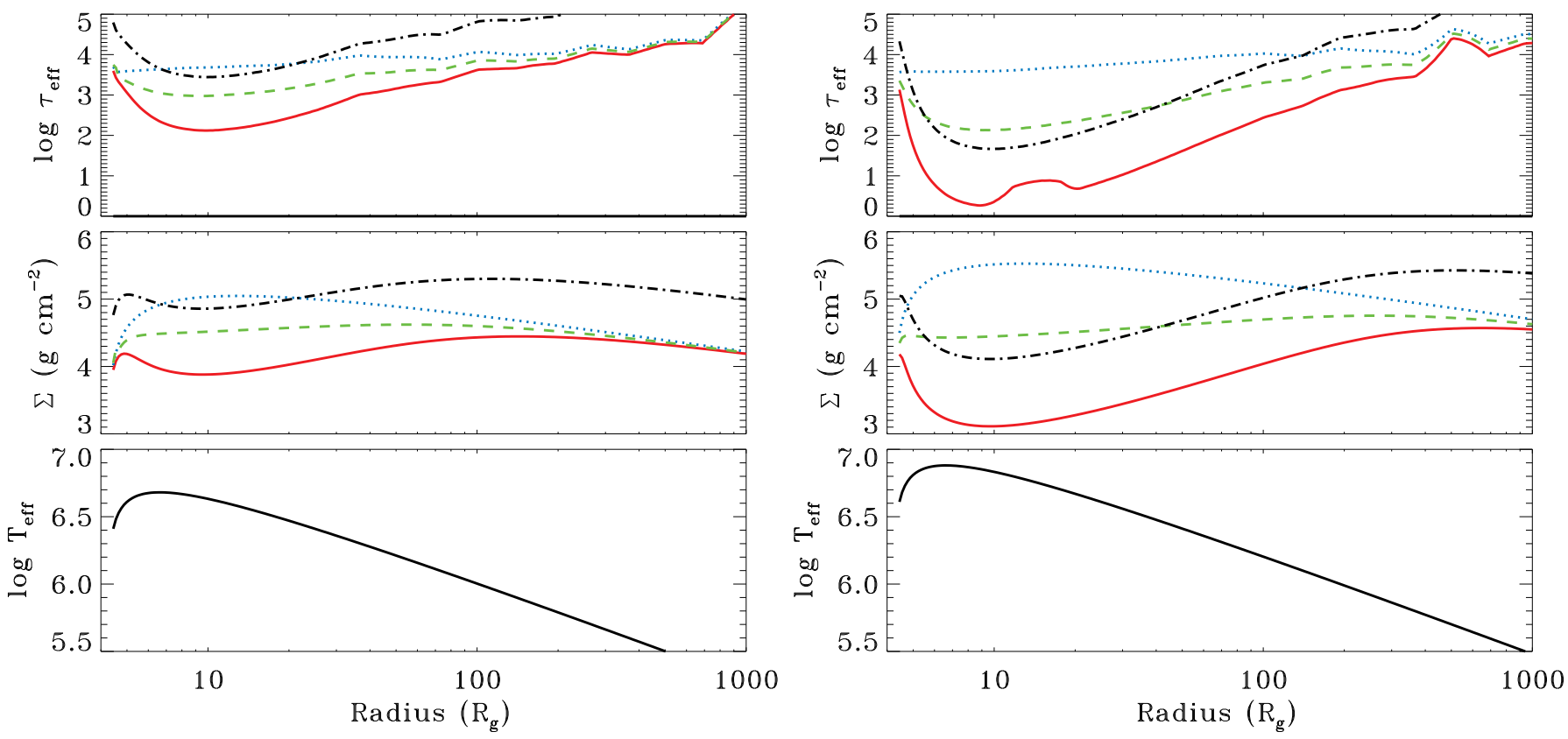

FIG. 2.- The upper, middle and lower panels show the effective optical depth to the disk midplane, total surface density and effective temperature as a function of radius, respectively. We compare alpha disks with $\alpha=0.01$ (black, dot-dashed) and $\alpha=0.1$ (red, solid), beta disks with $\alpha=0.1$ (blue, dotted), and mean disks with $\alpha=0.1$ (green, dashed). $T_{\text {eff }}$ is independent of stress prescription. (a) shows these disk parameters for $\log l=-1$ while (b) has $\log l=-0.2$.

and $\left\langle P_{\text {gas }}\right\rangle$ in terms of $\Sigma$, yielding two equations (2, 3) for two unknowns $(\Sigma, H)$. The resulting $\Sigma$ evolves smoothly between the gas and radiation pressure dominated limits. Previous work (Davis et al. 2005) has shown that the resulting estimates for $\Sigma$ typically agree with a more precise iterative method to $\lesssim 30 \%$.

Our results also agree with the gas and radiation pressure dominated limits which have been worked out in detail by previous authors (e.g. Shakura \& Sunvaev 1973; Stella \& Rosner 1984; Laor \& Netzer 1989; Merloni 2003). We give the asymptotic behavior at large radii (away from the inner boundary condition) below as this is useful in understanding the subsequent plots. For the gas pressure dominated limit all three stress prescriptions give identical results where

$$
\Sigma_{\text {gas }} \propto m^{1 / 5} l^{3 / 5} r^{-3 / 5} \alpha^{-4 / 5}
$$

where $l \equiv L / L_{\text {Edd }}$ is the Eddington ratio, $r \equiv R / R_{g}$ is radius in unit of the gravitational radius, and $m \equiv$ $M / M_{\odot}$ is the black hole mass. For the radiation pressure dominated limit the surface density is sensitive to the stress prescription, and for our choices gives

$$
\begin{aligned}
\Sigma_{\mathrm{rad}, \alpha} & \propto l^{-1} r^{3 / 2} \alpha^{-1}, \\
\Sigma_{\mathrm{rad}, \beta} & \propto m^{1 / 5} l^{3 / 5} r^{-3 / 5} \alpha^{-4 / 5}, \\
\Sigma_{\mathrm{rad}, \operatorname{mean}} & \propto m^{1 / 9} l^{-1 / 9} r^{1 / 3} \alpha^{-8 / 9} .
\end{aligned}
$$

Since the BHBs considered here cover only a small range in mass, the weak dependence of $\Sigma$ on $m$ can be ignored. In what follows, the Eddington ratio dependence will be most important. For the alpha and mean $\operatorname{disks} \Sigma$ decreases as $l$ increases, but with a much weaker dependence for the mean disk. In contrast, $\Sigma$ increases with $l$ in the beta disk model. The susceptibility of the stress prescriptions to viscous instability follows directly from these relations. The condition for stability is that $d \Sigma / d \dot{M}>0$ (Lightman \& Eardlev 1974). Since $\dot{M} \propto l$, we see that only the beta disk is truly stable in the limit where radiation pressure dominates entirely, though the mean disk is also very close to the stability condition.

\section{LUMINOSITY-TEMPERATURE PLOTS AND THE COLOR-TEMPERATURE CORRECTION}

We now use this bhspec code (Davis \& Hubeny 2006, see also \$2) to investigate the spectra produced by these different stress prescriptions for a black hole of $10 M_{\odot}$, inclined at $60^{\circ}$, with spin of $a_{*}=0.5$. We use the XSPEC spectral fitting package to convolve the models with the detector response from current X-ray instruments in order to simulate what would be observed. We do this for accretion rates equally spaced in $\log l$ from -1.4 to -0.2 through both the RXTE PCA (3-20 keV) and Swift XRT (0.1-10 keV) responses. These two instruments are broadly characteristic of any proportional counter (e.g. GINGA, ASTROSAT) and CCD (e.g. Chandra, XMMNewton, Suzaku XIS) responses, respectively. We fix the source distance at $8.5 \mathrm{kpc}$ (Galactic center), and assume a Galactic absorbing column of $N_{H}=1.2 \times 10^{21} \mathrm{~cm}^{-2}$ (approximately the lowest possible at this distance). We note that for $\log l=-0.6$, this gives a very good estimate of the disk dominated spectrum of XTE J1817-330 at the peak of its outburst (spectrum 001: Rykoff et al. 2007; Gierlinski et al. 2008, hereafter GDP08).

We also include a small Comptonized tail, with bolometric luminosity of $\sim 5 \%$ of that of the disk, with pho- 

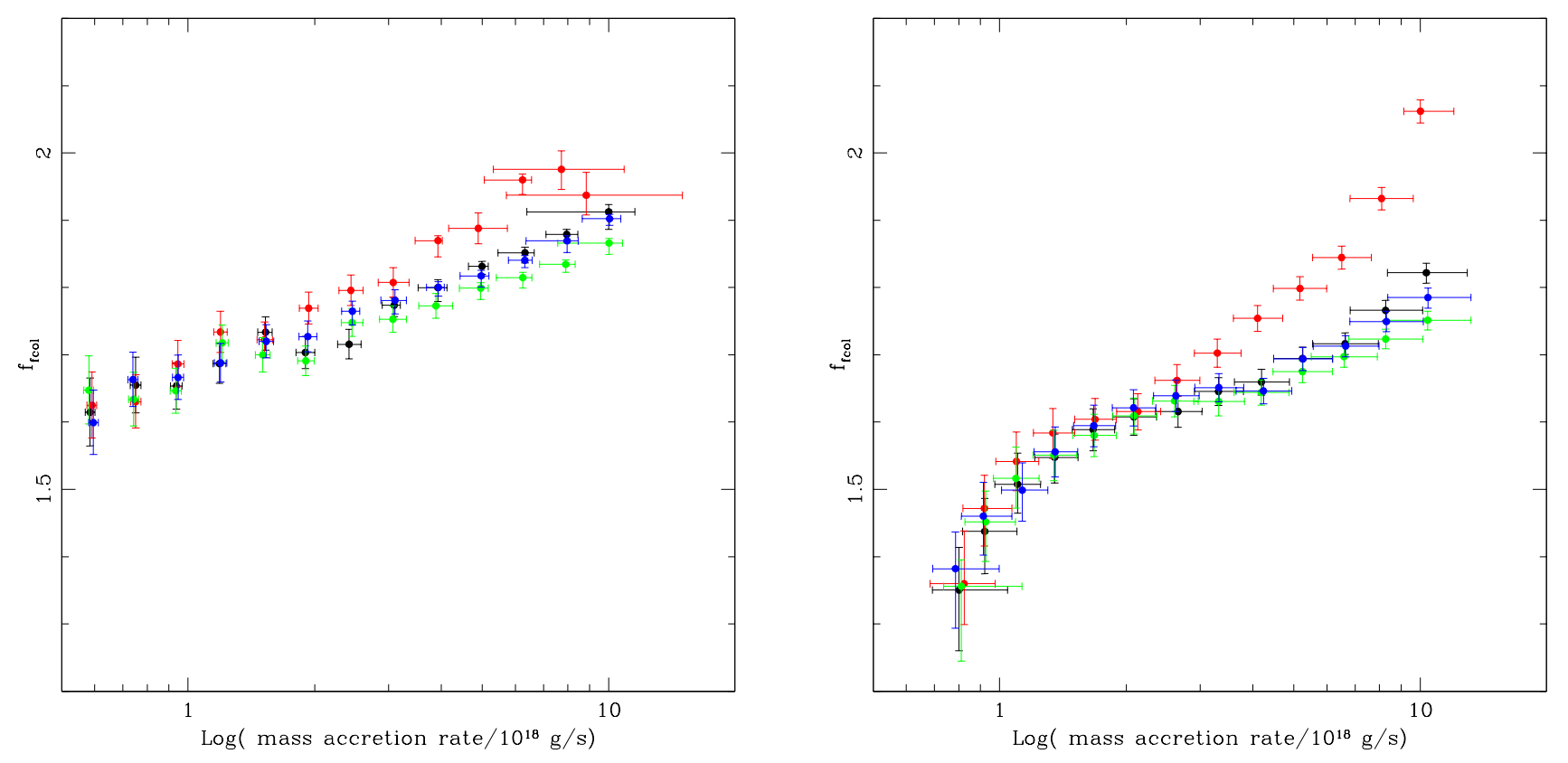

FIG. 3.- Color-temperature corrections from fits of bhspec with kerrbb as described in 3 The left panel corresponds to fits using a CCD (Swift XRT) response while the right panel is for a proportional counter (RXTE PCA) response. We consider four different stress prescriptions: alpha disks with $\alpha=0.01$ (black) and $\alpha=0.1$ (red), beta disks with $\alpha=0.1$ (blue), and mean disks with $\alpha=0.1$ (green).. Comparison with Fig 1 shows that the results for the color-temperature correction are not the same as for diskbb, where the CCD bandpass gives an almost constant $f_{\text {col }}=1.65-1.75$ as opposed to the change from 1.6-1.9 seen here. The proportional counter likewise has $f_{c o l}=1.7-2.0$ with diskbb compared to $1.4-1.8$ here.

ton index of $\Gamma=2.2$ and electron temperature fixed at $100 \mathrm{keV}$ (i.e. outside the bandpass of either instrument). The seed photons are assumed to be a blackbody at the maximum inner disk temperature, set by noting that disk fits to the peak spectrum of XTE J1817-330 give $k T_{\text {disk }} \sim 0.9 \mathrm{keV}$ (GDP08). We use this as our fiducial seed temperature for $\log l=-0.6$, and then change it by a factor $\left(10^{0.1}\right)^{1 / 4}=1.059$ for each 0.1 dex change in $\log l$. We also add $1 \%$ systematic uncertainties to our simulated PCA spectra as is usual with real data.

These simulated spectra are then fit with a very simple multicolor disk blackbody model, diskbb (Mitsuda et al. 1984). This assumes that the disk temperature $T(r) \propto$ $r^{-3 / 4}$, i.e. has continuous stress at the inner boundary. The emitting area can still be derived from the data by applying a correction factor for this (Kubota et al. 1998) as the overall shape of this spectrum is very similar to that produced by a stress-free inner boundary condition (Gierliński et al. 2001). We also include a Comptonized tail produced from (blackbody) seed photons tied to the inner disk temperature and absorption fixed at Galactic values. The disk so dominates the CCD bandpass that the Compton tail is poorly constrained, and allowing the spectral index to be free gives best fit parameters, for which the Comptonized tail is very steep. This is due to the bhspec disk shape being subtly different to a simple sum of blackbodies, firstly as relativistic effects smear out the Wien tail and secondly as the color-temperature correction is not constant as a function of radius. These combine to give a broader disk spectrum than predicted by diskbb so the low temperature Comptonization is required to extend the emission to higher energies. Instead, we make the assumption that simultaneous high energy data exist to constrain the spectral index (such as from an $R X T E$ monitoring campaign: e.g. GDP08), hence we fix this at the input value of 2.2 in the CCD fits, but allow it to be free in the proportional counter models.

Fig. 1 1 shows the resulting $L-T$ diagram from fitting the CCD and proportional counter simulations for the two new stress prescriptions considered here (green: beta disk with $\alpha=0.1$, blue: mean disk with $\alpha=0.1$ ), together with the two previous alpha disk models for comparison (black: $\alpha=0.01$ and red, $\alpha=0.1$ ). All the stress prescriptions which yield large surface density $(\alpha=0.01$, mean and beta disks) show very similar behavior. Only the alpha disk with $\alpha=0.1$ gives significantly higher temperatures at high luminosities. However, at low luminosities, all the stress prescriptions give a fairly good $T^{4}$ relation, showing clearly that the Merloni et al. (2000) result of a substantial increase in color temperature at low luminosities is indeed an artifact, and is not representative of a gas pressure dominated beta disk (c.f. the discussion in Gierliński \& Done 2004, where the Merloni et al. result was used to argue against a beta disk model).

The luminosity in these plots is derived as in the $L-T$ plots of GD04 and DGK07 by correcting the disk flux and temperature for inclination angle and relativistic effects. The general relativistic corrections are taken from Zhang et al. (1997) for a Schwarzschild black hole as this is closer to an $a_{*}=0.5$ disk than the alternative tabula- 

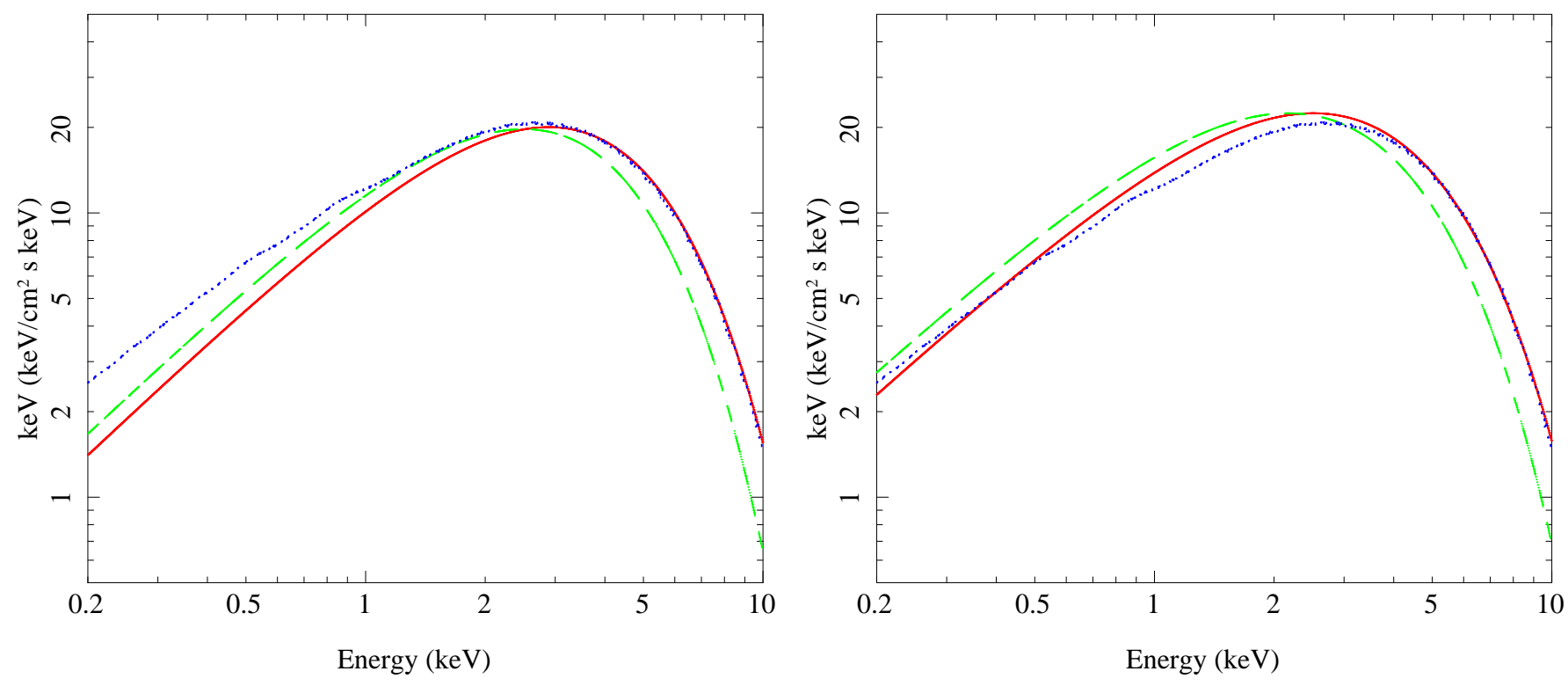

FIG. 4.- a) The best-fit diskbb models to simulated bhspec disk spectra with $a_{*}=0.5$ and log $l=-0.3$ for the proportional counter (red, solid curve) and CCD (green, dashed curve) responses. For comparison, we also plot actual bhspec model (blue, dotted curve) used to generate the simulated spectrum. The diskbb spectrum is narrower than bhspec so the best fit models adjust as the bandpass changes. The CCD gives a lower temperature by a factor 1.15, and the discrepancy at 5-10 keV is taken up by the freedom in fitting the Comptonized tail. b) A similar comparison of kerrbb models with the same bhspec model plotted in panel a. However, in this case the kerrbb spectra are not best fit models. Instead, they have spin and mass accretion rates set at the intrinsic parameters for the bhspec model, but with color-temperature corrections chosen to have the same ratio (1.15, see text for further details) as the best-fit diskbb temperatures. It is plain that the color-temperature corrections derived from the diskbb fits are not appropriate for kerrbb because the models have different spectral shapes.

tion for $a_{*}=0.998$. However, these have very little effect at $60^{\circ}$, as this is where the Doppler blueshift and gravitational redshifts approximately cancel, thus the flux to luminosity conversion is very close to a simple disk area correction of $L=2 \pi D^{2} F / \cos i=4 \pi D^{2} F$ for $\cos i=0.5$, and the temperature correction is negligible. However, the recovered flux is less than the input value (e.g. for the highest luminosity points made from $\log l=-0.2$, while the dense disks give -0.26) as limb darkening is present in the bhspec radiation transfer.

The solid lines show the predicted $L-T$ relation for a constant color temperature of 1.6, 1.8, 2.0 and 2.2 again as in GD04, but scaling the disk area to that expected from an $a_{*}=0.5$ black hole. Clearly the CCD data are very close to a constant value of $f_{\mathrm{col}}=1.7$, while the proportional counter bandpass gives a small change in $f_{\text {col from } 1.8-2.0}$ for the dense disks, and 1.8-2.2 for the alpha disk with $\alpha=0.1$.

The top panels of Fig. 2 show the effective optical depth of the disk $\left(\tau_{\text {eff }} \equiv \sqrt{\tau_{\text {abs }}\left(\tau_{\text {es }}+\tau_{\text {abs }}\right)}\right)$ as a function of radius for $\log l=-1$ and -0.2 respectively. For comparison we also plot the surface density (middle) and effective temperature (bottom) for the same models. For alpha disks the minimum in $\tau_{\text {eff }}$ closely corresponds to the minimum in $\Sigma$. Furthermore, these minima are close to the radius of maximum $T_{\text {eff }}$, so the majority of the flux will be produced in the regions of the disk which have the lowest opacity and hence the highest color temperature. Comparison of the top and middle panels show that $\tau_{\text {eff }}$ is very similar in shape to $\Sigma$. For a given alpha, the beta disk always yields a larger $\Sigma$ (and, therefore, a larger $\left.\tau_{\text {eff }}\right)$ than the alpha disk, with the mean disk midway between them. Due to the different scalings with $l$ (see Eqs. 4 and (5), the models become more discrepant as luminosity increases. The alpha disk with $\alpha=0.1$ (red, solid curve in Fig. 22) always has the lowest effective optical depth, and so gives the largest color-temperature correction. However, the effect of this is small at low luminosities (Fig. 1) as there is still substantial absorption opacity (Fig. 2a) and the heating mainly occurs at high effective optical depth. Thus, the photosphere acts simply as an atmosphere. By contrast, at the highest luminosities, the alpha disk with $\alpha=0.1$ approaches $\tau_{\text {eff }}=1$. All of the flux is dissipated above the effective photosphere itself, and the color-temperature correction becomes much larger.

We repeat the spectral fitting, replacing the simple diskbb model by the more physical kerrbb spectrum (Li et al. 2005). Like diskbb, kerrbb is a multicolor blackbody model, but it includes all of the relativistic effects modeled in bhspec i.e. all the special and general relativistic smearing of the intrinsic disk emission due to rapid rotation in strong gravity. These broaden the spectrum so that it is not so sharply peaked as predicted in the diskbb models, but unlike bhspec it assumes that the intrinsic emission from each radius is just a colortemperature corrected blackbody, and that this color correction factor is the same at all radii. Therefore, the derived $f_{\text {col }}$ will arise solely from differences in the treatment of the surface emission (see e.g. Shafee et al. 2006). We fix the mass, distance, inclination and spin, and fit for the mass accretion rate and color-temperature correction assuming no stress on the inner boundary and no returning radiation, but including limb darkening. Fig. 
3 shows these for the CCD (left panel) and proportional counter (right panel) data respectively. Again the three dense disk prescriptions all show similar results, with the alpha disk with $\alpha=0.1$ giving higher color-temperature correction at high luminosity, as before. However, the values of the derived color-temperature corrections are different to those derived from the diskbb approach. The color-temperature correction for kerrbb does not stay constant in the CCD bandpass, apparently in conflict with the observed $L \propto T^{4}$ relation from diskbb. It increases from 1.6-1.9, which at least goes through the $f_{\text {col }}=1.7$ constant value seen with diskbb. By contrast, the proportional counter bandpass gives values of the kerrbb color-temperature correction which are always lower than those seen from diskbb, as well as spanning a wider range, from 1.35-1.8

The answer to these issues lies in the detailed shape of the spectrum. Fig. 4a shows the initial bhspec disk (blue, dotted line) and its best fit diskbb spectra to the proportional counter (red, solid line) and CCD (green, dashed line) bandpasses for one of the dense disk prescriptions $(\alpha=0.01)$ for an input luminosity of $\log l=-0.3$. The higher energy bandpass of the proportional counter weights the fit to higher temperatures/lower normalization than those for the CCD, and the diskbb model is a good fit to the bhspec above the $3 \mathrm{keV}$ lower limit of the proportional counter bandpass. However, the best fit to the CCD data does not look so compelling. It is clear that the diskbb spectrum is narrower than the bhspec disk emission, and the 'best fit' matches this only in the 1-3 keV range where the signalto-noise is maximum. At higher energies, the Compton tail provides additional freedom to match the data.

Fig. 4b shows the kerrbb models with mass accretion rate chosen to give $\log l=-0.3$ for a black hole with $a_{*}=$ 0.5 (i.e. $8.537 \times 10^{18} \mathrm{~g} / \mathrm{s}$ ), with color-temperature correction of 1.7 for the proportional counter (red). The ratio of best fit temperatures from the diskbb models above implies that the color-temperature correction should be a factor 1.15 smaller in the CCD compared to the proportional counter i.e. giving $f_{\text {col }}=1.47$ for the CCD (blue). However, the kerrbb spectrum is much broader than diskbb as it includes the relativistic smearing of the continuum, and the proportional counter model is actually a fairly good description of the bhspec disk shape apart from around the peak at $\sim 1 \mathrm{keV}$. This is because bhspec includes the photoelectric edge features in the atmosphere, calculating the radiative transfer through the ion populations rather than simply assuming a diluted blackbody. These atomic features, from partially ionized oxygen/silicon/iron L shell, depress the continuum around $1 \mathrm{keV}$ from that predicted by the kerrbb models.

Thus the kerrbb model appropriate for the proportional counter data gives a good match below $\sim 0.6 \mathrm{keV}$ but overestimates the flux at the peak. Decreasing the color-temperature correction simply increases the disparity between the kerrbb and bhspec disk spectra in the CCD bandpass, especially in the crucial 1-3 keV region where the signal-to-noise is high. Thus the appropriate kerrbb model would have slightly higher colortemperature correction for the CCD than for the proportional counter, as observed (see Figs. $3 \mathrm{a}$ and b). These differences in spectral shape mean that colortemperature corrections derived from diskbb cannot be simply applied to kerrbb (and vice versa).

\section{COLOR-TEMPERATURE CORRECTION AND} EFFECTIVE OPTICAL DEPTH FOR ALL SPIN

We use the insights derived above from the particular case of $a_{*}=0.5$ to extend our analysis of $\tau_{\text {eff }}$ and $f_{\text {col }}$ to a larger range of black hole spins and luminosities. We are primarily interested the role $\tau_{\text {eff }}$ plays in determining $f_{\text {col }}$. Since our fitting procedure is most sensitive to the hottest annuli, we will focus only on $\tau_{\text {eff }}$ at the radius where $T_{\text {eff }}$ is maximum. For each $a_{*}$ and $l$ we calculate the radius of maximum $T_{\text {eff }}$, and evaluate $\tau_{\text {eff }}$ at the midplane for the four stress prescriptions considered in $\$ 3$, Fig. 5 shows contour plots of the effective optical depth at the radius of maximum flux (c.f. Fig. 2) as a function of spin and luminosity for four different stress prescriptions. The calculations span a range of $a_{*}$ from 0 to 0.8 and $\log l$ from -2 to 0 . (We consider higher spins below.)

For comparison, we also fit the kerrbb model to simulated bhspec spectra using the PCA and XRT responses. The procedure we follow is identical to that described in 93 , but we now calculate $f_{\text {col }}$ over the same range of $a_{*}$ and $l$ as in Fig. 5. Fig. 6 shows the resulting color-temperature corrections for kerrbb fit over a proportional counter (PCA, solid) and CCD (XRT, dashed) bandpass. (We stress again that the different shape of kerrbb to that of diskbb means that these are not applicable to diskbb fits.) Except for the highest luminosities of the alpha disk model with $\alpha=0.1$, the CCD response always yields a higher $f_{\text {col }}$ than the proportional counter.

A key issue which determines the spectrum is the fraction of energy dissipated in the region where thermalization will be incomplete i.e. above $\tau_{\text {eff }} \sim 3$. This crucial translucent region of the disk is simply an atmosphere (negligible dissipation) for very optically thick disks, as the heating occurs at much greater depths. However, when the whole disk has $\tau_{\text {eff }}<30$ then a substantial fraction of the energy can be emitted in the translucent region, leading to a large increase in $f_{\text {col }}$. Fig. 5 shows that the alpha disk with $\alpha=0.1$ (panel b) is the only prescription for which the majority of the flux can be produced in the region with $\tau_{\text {eff }}<3$ (i.e. $\log \tau_{\text {eff }}=0.5$ ). This happens only for luminosities near Eddington and is only weakly dependent on spin. A comparison with Fig. 6 shows that these models are also the ones with highest $f_{\text {col }}$. The other three stress prescriptions (alpha disks with $\alpha=0.01$, beta and mean disks with $\alpha=0.1$ ) tend to give more modest $f_{\text {col }}$ at these $l$. This comparison also identifies $\tau_{\text {eff }} \simeq 30$ as the point at which dissipation in the atmosphere becomes important. At lower luminosities $(\log l \lesssim-0.5) \tau_{\text {eff }}$ is larger and $f_{\text {col }}$ is rather insensitive to the stress prescription.

For these low-to-moderate spins $\left(a_{*} \leq 0.8\right)$ the variations of $f_{\text {col }}$ and $\tau_{\text {eff }}$ have a much stronger dependence on $l$ than on $a_{*}$. These spins are probably the ones most appropriate for the majority of BHB which have low mass companions, as there is not enough mass in the companion for the accreted material to significantly change the angular momentum of the black hole (King \& Kolb 1999). Thus they fairly accurately reflect the birth spin distribution from massive star collapse, for which the best estimates give spin $a_{*} \lesssim 0.75-0.9$ (Gammie et al. 2004). However, in the rarer high mass X-ray binaries (which probably also form the ULX population e.g. King 


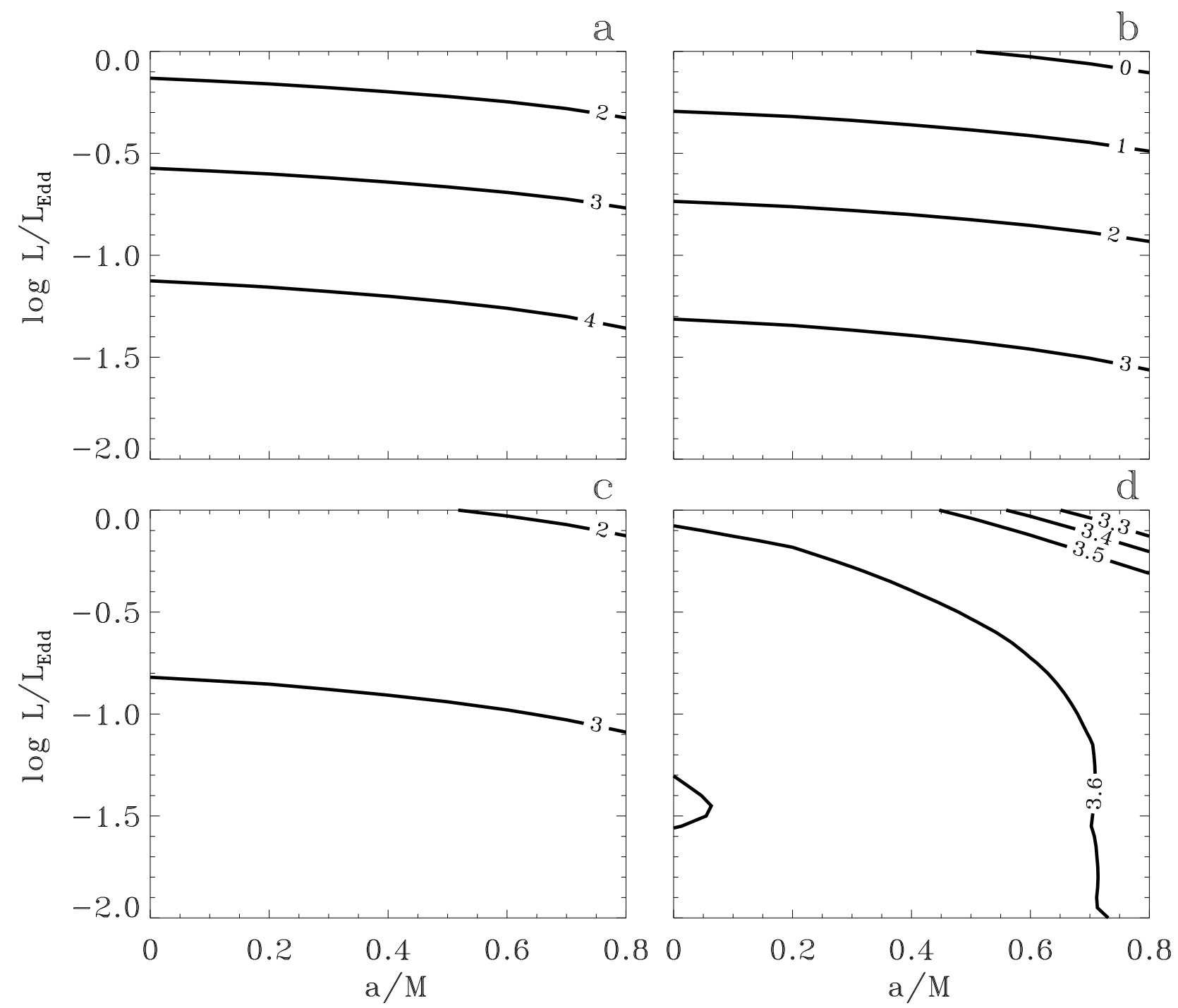

FIG. 5.- Effective optical depth at flux maximum as a function of spin and luminosity. The contour labels are values of log $\tau_{\text {eff }}$. The panels correspond to alpha disks with $\alpha=0.01$ (a) and $\alpha=0.1$ (b), mean disks with $\alpha=0.1$ (c), and beta disks with $\alpha=0.1$ (d). Note the change in scale of the contours for panel d. There is less than one order of magnitude change in effective optical depth across the whole range, so we decrease the spacing to 0.1 dex as opposed to 1 dex for the other panels.

2008) there is more mass in the companion star, so accretion may change the mass and spin of the black hole if the lifetime is long enough. Thus we also investigate higher spins, but these are computationally problematic for alpha disks with $\alpha=0.1$ as they become very effectively optically thin for $a_{*} \sim 1$ and $l \sim 1$. Since this stress prescription is ruled out by both spectral and variability behavior for the BHB, we take only alpha disks with $\alpha=0.01$ as a guide to the behavior of the other dense disks (mean and beta) as a function of spin for $0.8<a_{*} \leq 0.99$. Contour plots for $f_{\text {col }}$ and $\tau_{\text {eff }}$ are shown in Fig. 7]. The contours show a slightly stronger dependence on $a_{*}$ at these higher values, with $\tau_{\text {eff }}$ decreasing and $f_{\text {col }}$ increasing as $a_{*}$ approaches unity. Nonetheless, the overall effect is still relatively small compared to the much stronger dependence on $l$, and the conclusions drawn from Fig. 1 generalize quite broadly to other spins. Thus this predicts that any of the dense disk stress prescriptions will give disk spectra at high spin with $f_{\text {col }}$ increasing slightly with $l$ in a way which is not significantly different to that for low spin.

Thus extreme spins should simply manifest themselves as higher temperature disks at a given luminosity, giving a clear signature of a maximal Kerr black hole. However, none of the high mass X-ray binaries (e.g. Cyg X-1) show any signs of this, so we conclude that accretion has not yet had time to significantly spin up the black holes in these systems. The ULXs are also potentially high spin objects (Ebisawa et al. 2003; Hui \& Krolik 2008), but these have spectra which are often more complex than a simple disk model (see, however, Hui \& Krolik 2008), in which case they do not give a straightforward diagnostic of black hole mass and spin (Done \& Kubota 2006).

\section{DISCUSSION}

\subsection{Stress as a function of radius}


a
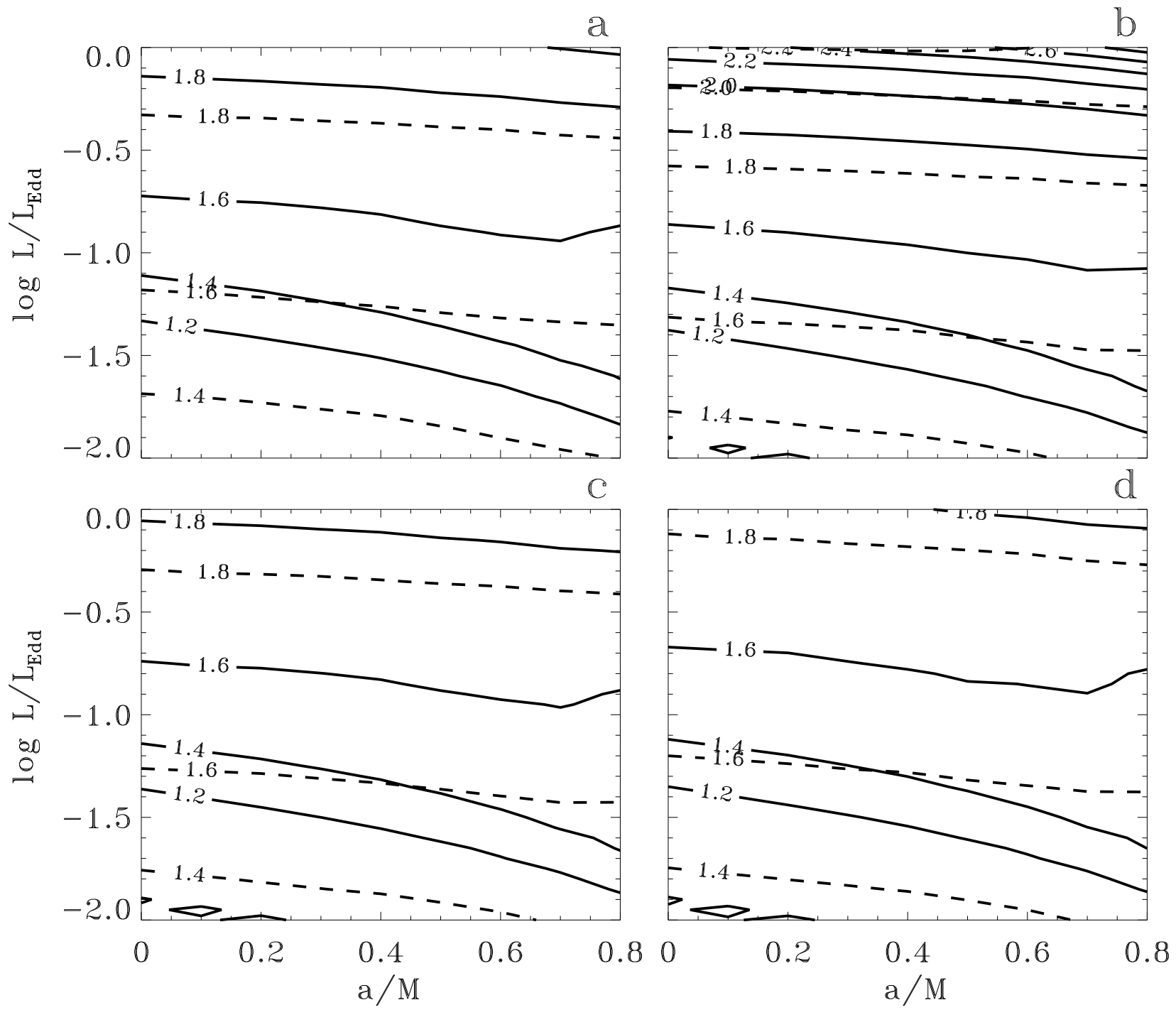

FIG. 6.- Color correction at flux maximum for kerrbb as a function of spin and luminosity. The solid and dashed contours are computed using the $R X T E$ PCA and Swift XRT responses, respectively. See text for more details. The panels correspond to different stress prescriptions as described in Fig. 5

The observation that stable disk spectra are seen spanning $l \sim 0.05-0.5$ shows that disks are not subject to the limit cycles predicted by the radiation pressure instability in this range. This rules out an alpha type stress, and instead requires that the surface density increases (or remains constant in the limit of marginal stability) as a function of $l$ at each radius, and this in itself implies that the accretion disk should remain effectively optically thick at high luminosities. Therefore the colortemperature corrections will be relatively constant, so predicting the approximate $L \propto T^{4}$ relation for the disk dominated spectra. The observation that most binaries show an approximate $L \propto T^{4}$ is strong confirmation of this conclusion.

Sadly, this also rules out the radiation pressure instability as being the physical mechanism for any observed behavior for $l<0.5$. This is otherwise an attractive possibility for the origin of the very high state, the other spectral state seen in binaries at high mass accretion rates. Although this does not show the predicted limit cycles, there is the possibility that the non-linear outcome of the instability could instead lead to strong Comptonization of the disk (e.g. Różańska \& Czerny 2000; Kubota \& Makishima 2004). Nonetheless, the arguments above preclude an alpha type stress, at least below $l \sim 0.5$, yet the very high state is indeed seen in the same range in luminosity as the disk dominated state (e.g. Remillard \& McClintock 2006).

At higher luminosities (up to $l \sim 3$, Done et al. 2004), there is a limit cycle observed in GRS1915+104 which could be the remnant of this radiation pressure instability from a marginally stable stress such as predicted with the mean disk (Honma et al. 1991; Merloni \& Navakshin 2006). However, this limit cycle behavior could alternatively be from other instabilities to do with the super Eddington flows. This is an important distinction, as the classic radiation pressure instability is not scale invariant with mass, but is triggered at $l \propto m^{-1 / 8}$. Thus even if the disk is stable in stellar mass black holes up to $l \sim 0.5$, it can be unstable at $l \gtrsim 0.09$ for a $10^{7} M_{\odot}$ 

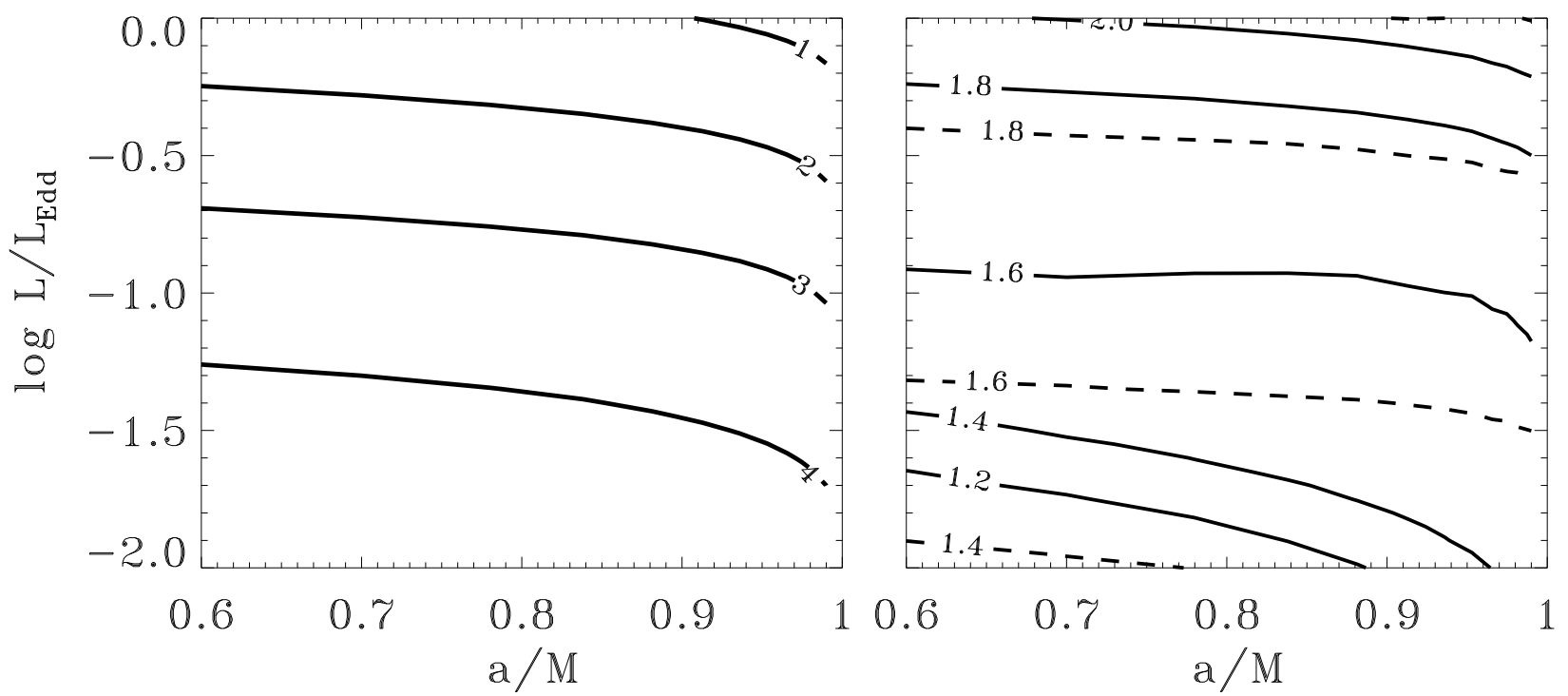

Fig. 7.- As in Fig. 5 but extended to high spin for an alpha disk with $\alpha=0.01$. The left panel gives effective optical depth at flux maximum, while the right panel gives the values of $f_{\text {col }}$ for kerrbb computed using the RXTE PCA (solid) and Swift XRT (dashed) responses, respectively.

AGN. In this case the instability could play a role in producing the puzzling, 'soft excess' seen in high mass accretion rate AGN, predominantly Narrow Line Seyfert 1's (Różańska \& Czernv 2000; Fabian et al. 2002). However, there are multiple similarities between the very high state and Narrow Line Seyfert 1 spectra to make it more likely that there is a similar explanation for both types of object.

Similarly, those properties of super Eddington flows which likewise depend on the alpha stress are also probably not a realistic description of these disks. Radiation trapping is a generic feature of any of the stress prescriptions (e.g. Honma et al. 1991), resulting in optically thick advection of energy especially in the disk midplane (Watarai et al. 2001). However, the denser disks which result from the alternative stress prescriptions are less likely to become effectively optically thin to the escaping radiation, so are unlikely to show the very large colortemperature correction which can arise from overheating of alpha disks (e.g. Beloborodov 1998; Kawaguchi 2003). We caution that fitting such models to high mass accretion rate spectra from ULX and NLS1's may not be appropriate.

\subsection{Caveats}

So what then are the caveats to using disk spectra as an estimator for black hole spin? The first is that the spectra must be disk dominated. The disk radius can be under - or overestimated when a substantial fraction of the dissipation goes instead into a corona, either in the very high (Kubota \& Done 2004; Done \& Kubota 2006) or low/hard state (GDP08). This may be the origin of the discrepancy in spin determination by disk spectral fitting in GRS 1915+104 (compare Middleton et al. 2006; McClintock et al. 2006). The spectra of GRS 1915+104 may well be more complex than the disk dominated spectra seen from BHB due to its higher luminosity (Done et al. 2004), perhaps powering strong winds (see below). It seems premature to apply the uncertainties from this one pathological object to other sub-Eddington BHB (e.g. Revnolds \& Fabian 2008).

Secondly, the value of the color-temperature correction is robust for all of the dense disk stress prescriptions, but varies with bandpass and also varies with the detailed disk model used to fit the spectra. We caution that differences in spectral shape between the models means that the same color-temperature correction factors cannot be simply applied to diskbb and kerrbb fits. Ironically, for both the generic proportional counter and CCD bandpasses, the diskbb fits give a simpler (approximately constant color temperature) representation of the data.

Thirdly, the structure of the disk as $l \rightarrow 1$ is not well modeled by this code. We neglect optically thick advection, and changes in the scale height of the disk with radius which may lead to self shielding for highly inclined objects (e.g. Watarai et al. 2005), but these are unlikely to have much effect for $l<0.5$, where the majority of BHB data are taken.

However, disks at high luminosities are likely to also power substantial winds. There are clear signs of an equatorial disk wind from observations of highly ionized, blueshifted, $\mathrm{Fe} \mathrm{K} \alpha$ absorption lines. These are ubiquitously seen in CCD data from high/soft state BHB (i.e. $l>0.1$ ) with inclination angles $\geq 60^{\circ}$ (e.g. DGK07). This material is probably a thermally driven disk wind, where irradiation of the outer disk layers produces a Compton heated skin whose high temperature allows gas particles to have enough energy to escape at large radii (Begelman et al. 1983; Woods et al. 1996), though this may be (substantially?) enhanced by magnetic fields (Miller et al. 2006). Such winds almost certainly become more powerful with $l$, especially as $l \rightarrow 1$ and radiation driving enhances them still further (Proga \& Kallman 2002). Counterintuitively, these stronger winds may become less observable as the stronger irradiation means that they become more ionized, losing even the He- and H-like Fe (Woods et al. 1996). Thus there may be winds which have substantial column, but are unobservable as 
they are completely ionized. Electron scattering has the effect of suppressing the observed luminosity by a factor $e^{-\tau}$ for lines of sight intersecting the wind, while the scattered flux $\left(1-e^{-\tau}\right)$ can enhance the luminosity along the disk axis by a factor $1+\left(1-e^{-\tau}\right) \Omega / 4 \pi$ where $\Omega$ is the solid angle subtended by the wind. This may be the origin of the occasionally observed 'bends' in the $L-T$ diagram, where the data deviate away from the generally observed slow increase in $f_{\text {col }}$ as a function of luminosity e.g. in GRO J1655-40 there is a much stronger increase in $f_{\text {col }}$ with $l$ than predicted by the dense disk models (e.g. Fig $1 \mathrm{~b}$ ), while in LMC X-3 there is a slight decrease (see e.g. Davis et al. 2006). While the inclination of LMC X-3 is not well known, GRO J1655-40 is plainly at very high inclination, and has clear wind features detected in multiple observations (Miller et al. 2006; Sala et al. 2007).

Thus as well as avoiding spectra with strong tails, and super Eddington sources, we also caution against any serious attempt to derive spin from 'bent' $L-T$ diagrams, where winds may be important.

Our results also hinge on the fact that the dissipation above the effective photosphere is small when the surface density is sufficiently large, so our assumptions about the dissipation profile with height may be an important caveat. Previous work (Davis et al. 2005; Blaes et al. 2006) has demonstrated that dissipation profiles motivated by radiative, stratified shearing box simulations (Turner 2004; Hirose et al. 2006) do not put enough dissipation near the surface to significantly alter the spectrum, although a more exhaustive range of parameter space needs to be explored. However, the alternative stress prescriptions discussed here are partially motivated by theoretical arguments that limit the magnetic stresses due to transport of buoyant magnetic field (see e.g. Stella \& Rosner 1984; Merloni 2003). If transport of magnetic field is significantly larger than currently seen in simulations, dissipation would be concentrated much nearer the surface than our models assume (Merloni 2003 ). This would give significant dissipation above the photosphere, and hence lead to a marked increase in color-temperature correction compared to those calculated here for the dense disks. As long as this is not a strong function of luminosity then it could still fit the data, but would lead to the spin being overestimated. Since the spins are generally low-to-moderate, there is not much scope for the real stresses to produce a significant fraction of the dissipation within the photosphere.

Similarly, the data also preclude a large amount of dissipation at the last stable orbit. If this were to thermalize it would lead to higher temperature emission, so current models would overestimate the spin, yet these already favor low spins. Alternatively, if the dissipation did not thermalize, it would instead produce a substantial nonthermal component in the spectrum, yet the spectra are dominated by the disk emission in the high/soft state. Thus there is only limited scope for stresses at the last stable orbit in disk dominated spectra, in contrast to the results from non-radiative, relativistic magnetohydrodynamic simulations (e.g. Beckwith et al. 2008). It may be that the character of the flow qualitatively changes when radiative cooling is included in the simulations, as the small scale height fields sustained by a thin (radiatively cooled) disk may lead to much less stress at the last stable orbit than the large scale fields which can be generated in the large scale height, non-radiative flows (Afshordi \& Paczyński 2003; Shafee et al. 2008).

This potential difference in dissipation between large scale height flows and thin disks may actually be observed in the behavior of the transition between the low/hard state, where the spectrum is produced by Compton scattering in a hot, optically flow, and the disk dominated state where the emission is quasi-thermal. The low/hard state is probably produced by a large scale height flow, which has an efficiency which is at least a factor 3 lower than that of a (stress-free inner boundary condition) thin disk down to the same radius (e.g. Naravan \& Yi 1995). This predicts that the luminosity at the transition should jump by at least a factor 3 , yet the data show a smooth transition of less than a factor $\sim 2$ (e.g. the compilation black hole binaries, especially Cyg X-1, in Done \& Gierliński 2004). This can be explained if the large scale height, less efficient flow in the low/hard state has continuous dissipation across the last stable orbit due to large scale height magnetic fields. In effect this allows the flow to extend down to smaller radii, so it taps a larger fraction of the gravitational potential energy, hence somewhat compensating for its lower radiative efficiency compared to that of the thin disk (Done et al. 2008, in preparation).

\section{CONCLUSIONS}

The detailed nature of angular momentum transport remains a significant uncertainty in our understanding of accretion flows and their emission. It is not yet entirely certain that the MRI, which is the best candidate for the source of the turbulent stress, truly does saturate at a level high enough to satisfy observational constraints (Pessah et al. 2007; Fromang \& Papaloizou 2007; King et al. 2007). Even if this question is resolved in favor of the MRI, a detailed understanding of the stress and resulting disk structure will likely require fully-relativistic, global simulations with realistic thermodynamics. Since such simulations require significant advances in computing power, we are forced rely on much simpler parameterizations of the stress until these become available. Even though these prescriptions are ad hoc, they may still capture important aspects of the physics in real astrophysical flows and provide useful constraints by direct comparison with observations.

With this motivation, we have computed the accretion disk spectra predicted by several different stress prescriptions using the most complete spectral code currently available. This includes both non-LTE ion populations, with radiative transfer in the disk and full general relativistic ray tracing to propagate this flux to the observer. These models give results which are very close to the observed behavior of the spectra of black hole binaries in the thermal dominant (high/soft) state from $L / L_{E d d} \sim 0.06 \rightarrow 0.6$. The majority of this data comes from proportional counters, and generally shows that the temperature changes slightly more rapidly with luminosity than expected for a disk of constant inner radius, and constant color-temperature correction (e.g. the compilation of DG04 and DGK07). This is as predicted by all the high surface density disk models presented here, and should hold generally for all stress prescriptions where less than 10 per cent of the energy is dissipated above the effective photosphere. The surface layers then sim- 
ply act like a passive atmosphere, with properties set by the effective temperature. The increase in temperature with luminosity gives rise to a small increase in colortemperature correction and the resulting spectra are remarkably similar irrespective of the detailed form of the stress.

Such model fitting can also place interesting constraints on the angular momentum transport. The classic alpha disk with $\alpha=0.1$ becomes effectively optically thin at the highest luminosities. The resulting increase in color temperature with luminosity is much more rapid than observed from existing data, ruling out such stress prescriptions. The standard alpha disk is also unstable to the thermal-viscous radiation pressure instability which predicts limit cycle behavior which is not observed, again showing that the disk is denser than such models predict. However, even these models give the same spectra at low luminosities $\left(L \lesssim 0.1 L_{\mathrm{Edd}}\right)$, where the surface density is sufficiently large for the disk to remain very effectively optically thick. Therefore, where we have a clear view of the disk, unaffected by a moderately optically thick disk wind, the disk spectra should provide a relatively robust estimator of the disk inner radius, and plausibly, the spin of the black hole.

We thank K. Beckwith, O. Blaes, M. Gierliński, I. Hubeny, J. Krolik, and A. Kubota for useful discussions. CD acknowledges support from a PPARC senior fellowship, and a Royal Society conference grant and Omer Blaes who together funded the visit to Santa Barbara at which this paper was begun. SD is supported by NASA grant number PF6-70045, awarded by the Chandra X-ray Center, which is operated by the Smithsonian Astrophysical Observatory for NASA under contract NAS8-03060.

\section{REFERENCES}

Afshordi, N., \& Paczyński, B. 2003, ApJ, 592, 354

Balbus, S. A., \& Hawley, J. F. 1991, ApJ, 376, 214

Beckwith, K., Hawley, J., \& Krolik, J. 2008, ArXiv e-prints, 801

Begelman, M. C., McKee, C. F., \& Shields, G. A. 1983, ApJ, 271, 70

Beloborodov, A. M. 1998, MNRAS, 297, 739

Blaes, O. M., Davis, S. W., Hirose, S., Krolik, J. H., \& Stone, J. M. 2006, ApJ, 645, 1402

Davis, S. W., Blaes, O. M., Hubeny, I., \& Turner, N. J. 2005, ApJ, 621,372

Davis, S. W., Done, C., \& Blaes, O. M. 2006, ApJ, 647, 525

Davis, S. W., \& Hubeny, I. 2006, ApJS, 164, 530

Done, C., \& Gierliński, M. 2004, Progress of Theoretical Physics Supplement, 155, 9

Done, C., Gierliński, M., \& Kubota, A. 2007, A\&A Rev., 15, 1

Done, C., \& Kubota, A. 2006, MNRAS, 371, 1216

Done, C., Wardziński, G., \& Gierliński, M. 2004, MNRAS, 349, 393

Eardley, D. M., \& Lightman, A. P. 1975, ApJ, 200, 187

Ebisawa, K., Makino, F., Mitsuda, K., Belloni, T., Cowley, A. P., Schmidtke, P. C., \& Treves, A. 1993, ApJ, 403, 684

Ebisawa, K., Mitsuda, K., \& Hanawa, T. 1991, ApJ, 367, 213

Ebisawa, K., Życki, P., Kubota, A., Mizuno, T., \& Watarai, K.-y. 2003, ApJ, 597, 780

Fabian, A. C., Ballantyne, D. R., Merloni, A., Vaughan, S., Iwasawa, K., \& Boller, T. 2002, MNRAS, 331, L35

Fromang, S., \& Papaloizou, J. 2007, A\&A, 476, 1113

Galeev, A. A., Rosner, R., \& Vaiana, G. S. 1979, ApJ, 229, 318

Gammie, C. F., Shapiro, S. L., \& McKinney, J. C. 2004, ApJ, 602, 312

Gierliński, M., \& Done, C. 2004, MNRAS, 347, 885

Gierlinski, M., Done, C., \& Page, K. 2008, ArXiv e-prints, 803

Gierliński, M., Maciołek-Niedźwiecki, A., \& Ebisawa, K. 2001, MNRAS, 325, 1253

Hirose, S., Krolik, J. H., \& Stone, J. M. 2006, ApJ, 640, 901

Honma, F., Kato, S., \& Matsumoto, R. 1991, PASJ, 43, 147

Hubeny, I., \& Lanz, T. 1995, ApJ, 439, 875

Hui, Y., \& Krolik, J. H. 2008, ArXiv e-prints, 803

Ichimaru, S. 1977, ApJ, 214, 840

Kato, S., Fukue, J., \& Mineshige, S., eds. 1998, Black-hole accretion disks

Kawaguchi, T. 2003, ApJ, 593, 69

King, A. R. 2008, MNRAS, 385, L113

King, A. R., \& Kolb, U. 1999, MNRAS, 305, 654

King, A. R., Pringle, J. E., \& Livio, M. 2007, MNRAS, 376, 1740

Kubota, A., \& Done, C. 2004, MNRAS, 353, 980

Kubota, A., \& Makishima, K. 2004, ApJ, 601, 428

Kubota, A., Makishima, K., \& Ebisawa, K. 2001, ApJ, 560, L147

Kubota, A., Tanaka, Y., Makishima, K., Ueda, Y., Dotani, T., Inoue, H., \& Yamaoka, K. 1998, PASJ, 50, 667
Laor, A., \& Netzer, H. 1989, MNRAS, 238, 897

Lasota, J.-P. 2001, New Astronomy Review, 45, 449

Li, L.-X., Zimmerman, E. R., Narayan, R., \& McClintock, J. E. 2005, ApJS, 157, 335

Lightman, A. P. 1974, ApJ, 194, 419

Lightman, A. P., \& Eardley, D. M. 1974, ApJ, 187, L1+

McClintock, J. E., Shafee, R., Narayan, R., Remillard, R. A., Davis, S. W., \& Li, L.-X. 2006, ApJ, 652, 518

Merloni, A. 2003, MNRAS, 341, 1051

Merloni, A., Fabian, A. C., \& Ross, R. R. 2000, MNRAS, 313, 193

Merloni, A., \& Nayakshin, S. 2006, MNRAS, 372, 728

Middleton, M., Done, C., Gierliński, M., \& Davis, S. W. 2006, MNRAS, 373, 1004

Miller, J. M., Raymond, J., Fabian, A., Steeghs, D., Homan, J., Reynolds, C., van der Klis, M., \& Wijnands, R. 2006, Nature, 441,953

Mitsuda, K., et al. 1984, PASJ, 36, 741

Narayan, R., \& Yi, I. 1995, ApJ, 452, 710

Nowak, M. A. 1995, PASP, 107, 1207

Pessah, M. E., Chan, C.-k., \& Psaltis, D. 2007, ApJ, 668, L51

Proga, D., \& Kallman, T. R. 2002, ApJ, 565, 455

Remillard, R. A., \& McClintock, J. E. 2006, ARA\&A, 44, 49

Reynolds, C. S., \& Fabian, A. C. 2008, ApJ, 675, 1048

Riffert, H., \& Herold, H. 1995, ApJ, 450, 508

Różańska, A., \& Czerny, B. 2000, A\&A, 360, 1170

Rykoff, E. S., Miller, J. M., Steeghs, D., \& Torres, M. A. P. 2007, ApJ, 666, 1129

Sakimoto, P. J., \& Coroniti, F. V. 1981, ApJ, 247, 19

Sala, G., Greiner, J., Vink, J., Haberl, F., Kendziorra, E., \& Zhang, X. L. 2007, A\&A, 461, 1049

Shafee, R., McClintock, J. E., Narayan, R., Davis, S. W., Li, L.-X., \& Remillard, R. A. 2006, ApJ, 636, L113

Shafee, R., Narayan, R., \& McClintock, J. E. 2008, ApJ, 676, 549

Shakura, N. I., \& Sunyaev, R. A. 1973, A\&A, 24, 337

一. 1976, MNRAS, 175, 613

Shimura, T., \& Takahara, F. 1995, ApJ, 445, 780

Stella, L., \& Rosner, R. 1984, ApJ, 277, 312

Taam, R. E., \& Lin, D. N. C. 1984, ApJ, 287, 761

Turner, N. J. 2004, ApJ, 605, L45

Watarai, K.-Y., Mizuno, T., \& Mineshige, S. 2001, ApJ, 549, L77

Watarai, K.-Y., Takahashi, R., \& Fukue, J. 2005, PASJ, 57, 827

Woods, D. T., Klein, R. I., Castor, J. I., McKee, C. F., \& Bell, J. B. 1996, ApJ, 461, 767

Zhang, S. N., Cui, W., \& Chen, W. 1997, ApJ, 482, L155+ 